\title{
Colorimetric gas sensors for RFID-Applications
}

\author{
Carolin Pannek ${ }^{1}$, Katrin Schmitt ${ }^{1}$, Ina Schumacher ${ }^{1}$, Sven Rademacher ${ }^{1}$, Jürgen Wöllenstein ${ }^{1,2}$ \\ ${ }^{1}$ Fraunhofer Institute for Physical Measurement Techniques, Heidenhofstr. 8, 79110 Freiburg \\ carolin.pannek@ipm.fraunhofer.de \\ ${ }^{2}$ Albert Ludwigs University Freiburg, IMTEK, Georges-Koehler-Allee 102, \\ 79110 Freiburg im Breisgau
}

\begin{abstract}
:
RFID offers the possibility, next to the identification, to monitor and/or control logistic processes e.g. for Food Chain Management. Here we present our investigations on the development and integration of colorimetric gas sensors on a RFID-platform. The reaction of a colorimetric sensor to its target gas leads to changes of its color, which is related to the target gas concentration. We developed different color dyes for $\mathrm{CO}, \mathrm{NO}_{2}$ and $\mathrm{NH}_{3}$ and utilize them as a sensitive layer in optical sensors. Absorption changes are detected by attenuation of the guided light. An optical waveguide is mounted on top of the RFID tag, together with a LED as a light source and a photo detector. The complete sensor system has a very low power consumption of $100 \mathrm{~mW}$ maximum.
\end{abstract}

Key words: RFID technology, colorimetric gas sensor, optical sensor, low-power sensors

\section{Introduction}

Many industries are focusing increasingly on the use of RFID technology (RFID: Radio Frequency IDentification) to monitor and control their processes optimally. RFID in combination with sensor technology (mainly temperature sensors) is already strongly used by the logistics industry and the food industry [1, 2]. The logistics industry puts an increasing emphasis on proper documentation of the transport routes of e.g. temperature sensitive goods. Here, the transport of vaccines plays an important role, as well as the transport and storage of perishable food. Even small increases in temperature promote the growth of harmful microorganisms, which makes temperature control highly important. For the period between production and delivery to the customer there is an increasing need for maintaining traceability. The identification, using RFID technology and the information of the sensors can be easily retrieved anywhere, just in time and controlled at every point of the transport route [3].

\section{RFID technology}

For many years, RFID technology has been used as a technical media to control and monitor logistic processes.

RFID communication is always between a reader and a transponder. The reader generates an electric field with the carrier frequency. Inductive coupling induces a voltage at the RFID antenna on the tag. After rectifying it can be used as power supply for a controller on the tag. This is called passive RFID communication. By adding a battery on the tag, the integration of low-power sensors is possible. The data can be stored actively on the tag until they are read out by the external electric field. These tags are called semi-active.

The historical development of RFID technology started at lower transmissions frequencies between $125 \mathrm{kHz}$ and $135 \mathrm{kHz}$, so-called LFRFID (low frequency). Due to the immunity of this transmission range to humidity and metal, LF-RFID is widely used e.g. as access control in buildings or as earmarks to identify animals etc [4]. LF transponders transmit unidirectional, which only allows the read-out of the transponder chip, but not the specific writing of information. Due to this working mode, only information about the identification, like ID numbers, can be read-out. A combination of LF-RFID with integrated sensors is thus not useful.

At higher frequency ranges (HF range), RFID transponders communicate at $13.56 \mathrm{MHz}$. This communication is by now established in different fields of application, e.g. as smartcards. Since the standardization of RFID communication in 2001, all HF-RFID systems communicate according to the ISO 15693 standard. ISO 15693 contains the physical parameters, the signal interface as well as the 
transmission protocol. The transmission range of HF-RFID systems is limited to a few $10 \mathrm{~cm}$. The integration of battery on HF-RFID systems enables the integration of low-power sensors.

To reach longer transmission ranges, UHFRFID systems use higher frequencies at 868 $\mathrm{MHz}$ in Europe, and $918 \mathrm{MHz}$ in the US. Nowadays these transponders are used in logistics for tracking and tracing of goods. The EPCglobal protocol is considered as standard for UHF communication. According to this protocol, the GS1 distributes unique ID numbers, which are like digital barcodes [5].

Table 1 gives an overview of the three different transmission frequencies and their typical parameters.

Tab. 1: Overview of the different RFID communication frequencies and their properties [4-6].

\begin{tabular}{|c|c|c|c|}
\hline & LF & HF & UHF \\
\hline Frequency & $<135 \mathrm{kHz}$ & $13,56 \mathrm{MHz}$ & $868 \mathrm{MHz}$ \\
\hline $\begin{array}{l}\text { Range } \\
(\max )\end{array}$ & $30 \mathrm{~cm}$ & $150 \mathrm{~cm}$ & $3.5 \mathrm{~m}$ \\
\hline $\begin{array}{l}\text { Influence } \\
\text { of humidity }\end{array}$ & no & no & $\begin{array}{l}\text { negative } \\
\text { influence }\end{array}$ \\
\hline $\begin{array}{l}\text { Influence } \\
\text { of metal }\end{array}$ & small & $\begin{array}{l}\text { negative } \\
\text { influence }\end{array}$ & $\begin{array}{c}\text { negative } \\
\text { influence }\end{array}$ \\
\hline Standard & $\begin{array}{c}\text { ISO } \\
11784 / 85 \\
\text { ISO } \\
18000-1\end{array}$ & $\begin{array}{l}\text { ISO } 15693 \\
\text { ISO } 14443\end{array}$ & $\begin{array}{l}\text { EPC GEN2 } \\
\text { ISO } 18000-6\end{array}$ \\
\hline
\end{tabular}

\section{Colorimetric Gas Sensing}

Working in combination with RFID technology means to support low-power and low-cost sensing. It was our aim to develop a simple gas sensing set-up using gasochromic effects. Colorimetric gas sensors are based on the change in color of a chemochromic reagent incorporated in a porous matrix. To implement such a sensor into a RFID system, the sensor has to work with ultra low-power consumption. In many applications, the integration of gas sensors on RFID tags is useful, e.g. the control of perishable food, luxury goods or sensitive medications.

The combination of standard gas sensors and RFID tags is demanding due to the high power consumption of such sensors. Low-cost sensors like metal oxide sensors (MOX sensors) have to be heated up to operation temperatures above $350^{\circ} \mathrm{C}$, which means several milliwatts of heating power. From our point of view, colorimetric gas sensors represent a proven alternative, thanks to their low power consumption.
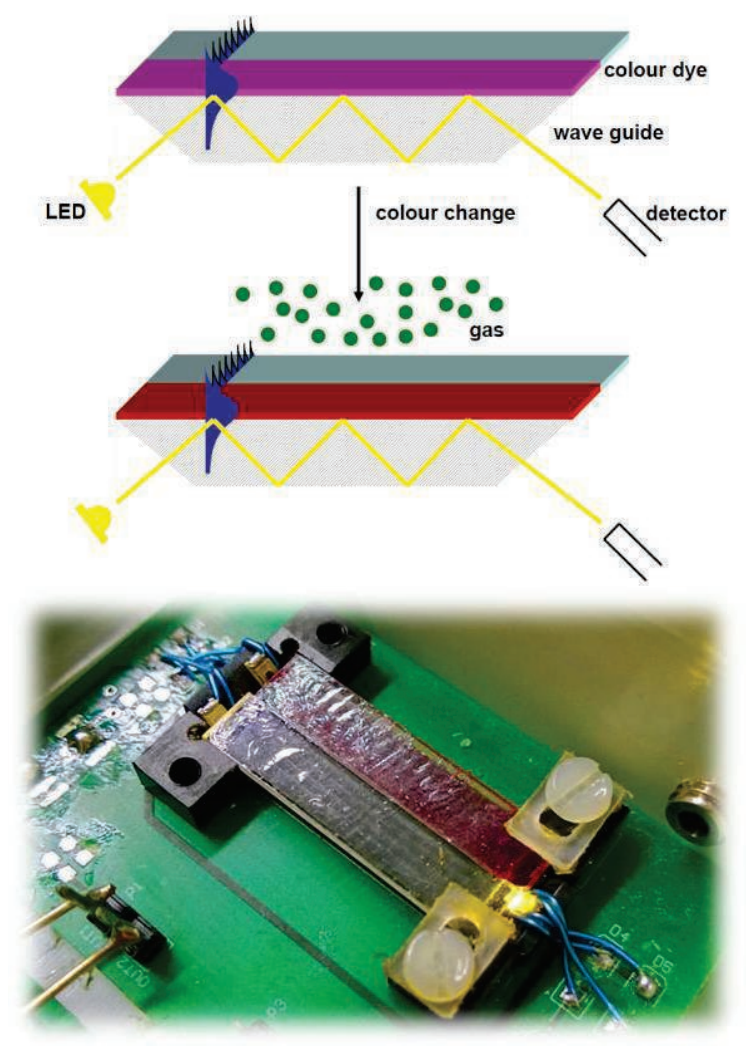

Figure 1. Above: Sketch of the gasochromic sensor consisting of a microscope slide with the lower half covered with the chemochromic film. The light of an $L E D$ is coupled into one end of the waveguide and travels trough it under TIR before it is focused onto a photo detector. The gas reaction leads to a color change of the color dye. Down: Picture of the realized sensor system.

The sensor set-up is based on a planar optical waveguide. In the easiest case, this can be the RFID label directly. This principle was already shown in $[7,8]$. Our approach is the integration of a glass slide onto which the dye is deposited. To obtain a planar and stable dye, the gasochromic material is embedded into a polymer matrix and deposited on the waveguide. These polymers should offer the following properties: long term stability, no swelling due to humidity and no interference with the dye. Possible polymers are poly vinyl chloride (PVC), polydimethylsiloxan (PDMS), ethyl cellulose (EC) and/or poly methylmethacrylate (PMMA).

Depending on the target gas, different color dyes can be embedded into the polymer matrix. Table 2 gives an overview of different dyes for ammonia, carbon monoxide and nitrogen 
dioxide. Their reaction to the gases has already been described in [9].

Tab. 1: Overview of gasochromic materials for $\mathrm{CO}$, $\mathrm{NO}_{2}$, and $\mathrm{NH}_{3}$ detection [7-9].

\begin{tabular}{|c|c|}
\hline & Parameters \\
\hline \multirow[t]{2}{*}{ Color dyes } & $\begin{array}{l}\text { CO: metal complex with transition } \\
\text { metal ion, e.g. binuclear rhodium } \\
\text { complexes (RhComp) [Litertur] } \\
\mathrm{NO}_{2} \text { : Chinonime color dyes like } \\
\mathrm{N}, \mathrm{N}, \mathrm{N}^{\prime}, \mathrm{N}^{\prime} \text { teramethyl-p- } \\
\text { phenylenediamine (TMPD, wursters } \\
\text { blue) or diphenylbenzidin (DBB) }\end{array}$ \\
\hline & $\begin{array}{l}\mathrm{NH}_{3} \text { : pH-indicators like bromphenol } \\
\text { blue (BPB), bromcresol green (BKG) } \\
\text { or methy red (MR) }\end{array}$ \\
\hline Color change & $\begin{array}{l}\mathrm{CO} \text { (RhComp): violet to yellow } \\
\mathrm{NO}_{2} \text { (TMPD): brown to blue } \\
\mathrm{NH}_{3} \text { (BPB): orange to blue }\end{array}$ \\
\hline$\lambda(\max )$ & $\begin{array}{l}\mathrm{CO} \text { (RhComp): } 480 \mathrm{~nm} \\
\mathrm{NO}_{2} \text { (TMPD): } 460 \mathrm{~nm} \\
\mathrm{NH}_{3} \text { (BPB): } 560-630 \mathrm{~nm}\end{array}$ \\
\hline $\begin{array}{l}\text { Measurement } \\
\text { range }\end{array}$ & $\begin{array}{l}\mathrm{CO}: 10-1.000 \mathrm{ppm} \\
\mathrm{NO}_{2}: 100 \mathrm{ppb}-10 \mathrm{ppm} \\
\mathrm{NH}_{3}: 0,5-50 \mathrm{ppm}\end{array}$ \\
\hline $\begin{array}{l}\text { Cross } \\
\text { sensitivities }\end{array}$ & $\begin{array}{l}\mathrm{CO}: \mathrm{NO}_{2}>10 \mathrm{ppm} \\
\mathrm{NO}_{2}: \text { no } \\
\mathrm{NH}_{3}: \text { no }\end{array}$ \\
\hline $\begin{array}{l}\text { Suitable } \\
\text { polymer }\end{array}$ & $\begin{array}{l}\mathrm{CO} \text { : ethyl cellulose } \\
\mathrm{NO}_{2} \text { : poly vinyl chloride } \\
\mathrm{NH}_{3} \text { : ethyl cellulose }\end{array}$ \\
\hline
\end{tabular}

The liquid polymer/color dye matrix can be deposited onto this waveguide by dip- or spin coating. The light of an LED is coupled into one end of the waveguide and passes through it under the conditions of total internal reflection (TIR). The color change of the sensitive dye can be detected by absorption changes inside the layer, and by changes in the evanescent field. After coupling out, the light is focused on a photo detector. Changes in the dye induce direct changes in the intensity of the light. Compared to measurements in transmission, the waveguide principle has much lower detection limits, caused by the longer optical path due to TIR. The color of the LED depends on the spectral section of the color change of the dye, cf. table 2. To be independent from environmental influences, a reference channel is embedded additionally.

As mentioned before, the senor only needs low power for operation. In case of a color-, i.e. signal change the sensor has a power consumption of about $100 \mathrm{~mW}$ maximum, with a settling time of max. $20 \mathrm{~ms}$. To obtain reliable measurements it is sufficient to switch on the LED for $100 \mathrm{~ms}$.

To improve the sensitivity of the sensor in case of small output signals, the signal-to-noise ratio can be enhanced by the use of a lock-in amplifier.

A scheme of the sensor set-up and a photo of the prototype are shown in figure 1 .

\section{RFID sensor tag}

The RFID sensor transponder platform consists of the RFID transponder chip, antenna battery, a $\mu$-controller and the colorimetric sensor. The antenna can be directly integrated on the board as an electronic circuit, depending on the transmission frequency, the shape of the antenna has to be adjusted. Figure 2 depicts the components of the RFID system with reader, reader antenna and transponder. The tag is set up as a semi-active HF transponder in the $13.56 \mathrm{MHz}$ band, fully compliant with the ISO15693 standard. The electronic circuitry of the gas sensor controls the measurements and allows communication with a PC, both with the lowest possible power consumption. For RFID communication, a commercially available RFID reader (Scemtec, Germany) was used and the corresponding transponder developed and integrated on the gas sensor system.

The classical RFID communication and data read-out is passive (load modulation) whereas the sensing and control electronics are active (in order to sense when the tag is out of the reading range).

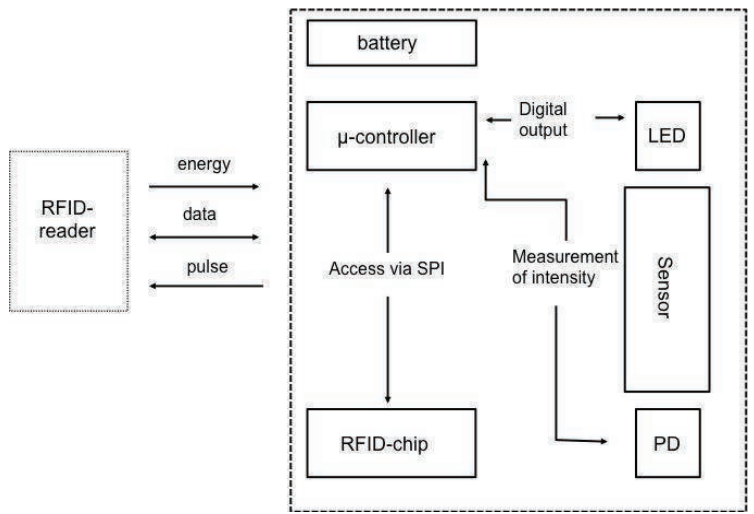

Figure 2. Block diagram of a RFIDsystem: the key component is the microcontroller, controlling the $L E D$ and reading the photodiode. The same controller communicates with the PC via the RFID chip.

As RFID-frontend we integrated a Melexis MLX90129 transponder chip. To date, only a few integrated (packaged) circuitries are freely available for RFID protocol implementation, 
MLX90129 is one of it. It can be controlled via an SPI interface, and comes with a 4096bit internal EEPROM (Electrically Erasable Programmable Read-Only Memory), which can be accessed either via SPI or via RFID. The data transfer between PC and microcontroller is accomplished using the EEPROM. The chip also contains a high-frequency module for radio transmission. As external circuitry only a suitable antenna is needed, this can be integrated as conductor line on the circuit board.

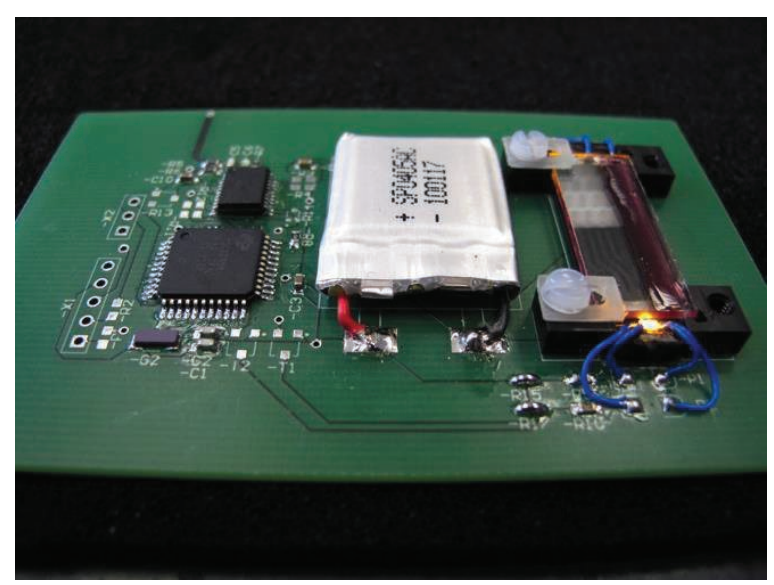

Figure 3. Picture of the realized RFIDsensor tag, with integrated gas sensor, RFIDtransponder chip, battery, $\mu$-Controller and EEPROM. The antenna for data transmission is realized on the backside.

For the implementation of the RFID protocol an adapted circuitry was used between the microcontroller and the SPI interface. The microcontroller also triggers and reads the LED and $P D$, thereby measuring the present gas concentration.

The sensor is mounted in a special holder that allows the arrangement of the LED and the photo detector in a $45^{\circ}$ angle for an improved light coupling. A picture of the realized RFIDsystem can be seen in figure 3 .

\section{Gas Measurements}

A series of different gas measurements was performed to evaluate the characteristics of the developed RFID-gas sensor tag.

Figure 4 shows the reaction of the CO-sensitive dye to $75 \mathrm{ppm} \mathrm{CO}$. The measurement was carried out in synthetic air with $40 \%$ r.H. The measurement shows the color change as a function of changes in the output voltage of the photodiode. After gas exposure, it takes several hours until the sensor reaches its baseline again.

The gas dependent reaction of BPB is shown in figure 5 . The sensor was exposed to different concentrations of $\mathrm{NH}_{3}$ between 1 ppm and 10 ppm. The measurement was performed in synthetic air with $40 \%$ r.H.. After the first three different gas concentrations the sensor was exposed to smaller steps of $\mathrm{NH}_{3}$. This measurement demonstrates the high reversibility of this color dye and, compared to $\mathrm{CO}$, the faster response time.

The received data of the photodiodes was stored in the EEPROM (one measured value each minute) and read out after the complete measurement cycle.

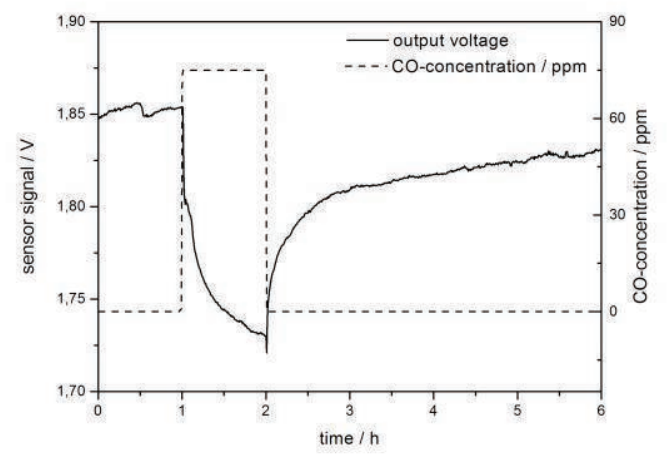

Figure 4. Gasochromic reaction of the CO-sensitive color dye [Literatur!!!] to $75 \mathrm{ppm}$ CO in synthetic air with $40 \%$ r.H.

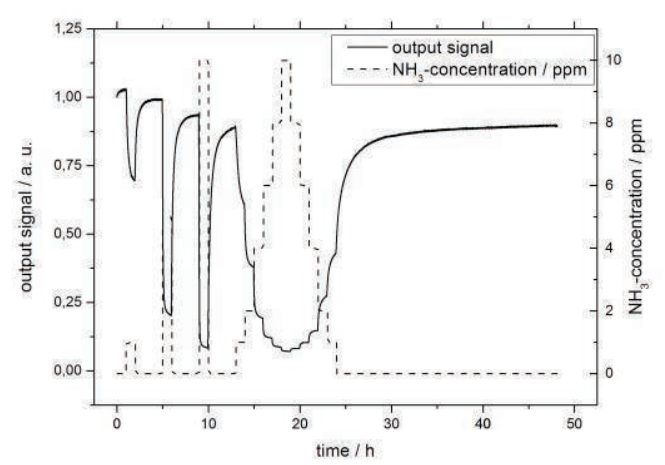

Figure 5. Gasochromic reaction of the $\mathrm{NH}_{3}$-sensitive color dye (BPB) to different $\mathrm{NH}_{3}$ concentrations between $1 \mathrm{ppm}$ and $10 \mathrm{ppm}$ in synthetic air with $40 \%$ r.H.

\section{Conclusion and Outlook}

In this paper we have shown the development of a RFID sensor tag with integrated gasochromic sensors for the detection of $\mathrm{NH}_{3}$, and $\mathrm{CO}$. The sensor is combined with an electronic circuitry for measurement control and RFID communication. The sensor was operated in a completely autonomous mode, i.e. battery driven, and the stored measurement results could be read via RFID.

The system was evaluated by a series of different gas measurements. The properties of the chemochromic films are summarized. 
Further work will focus on the integration of the color dye onto the sensor tag. The mounting used to date is not suitable for RFID label production. Ideally, the sensitive layer should be directly deposited by screen-printing on top of the label.

\section{Achnowledgement}

We like to thank the German BMBF and MST BW for funding main parts of the work within the project SensRFID, which is part of the LeadingEdge Cluster MicroTec Südwest ("Spitzencluster funding of the Federal Republic of Germany"). Furthermore we like to thank our industry partner Scemtec Transponder Technology in Gummersbach, Germany.

\section{References}

[1] E. Prater, G.V. Frazier, Future Impacts of RFID on e-supply chains in grocery retailing, Supply Chain Management: An International Journal, 10/2, 134-142 (2005); doi: $10.1108 / 13598540510589205$

[2] R. Jedermann, W. Lang, Semi-passive RFID and beyond: steps towards automated quality tracing in the food chain, International Journal of Radio Frequency Indentification Technology and Applications, Vol. 1, No. 3, 247-259 (2007)

[3] M. Attaran, RFID: an enabler of supply chain operations, Supply Chain Management: an International Journal, Vol. 12 Iss 4 (2007), 249257, DOI: 10.1108/13598540710759763

[4] C. Sprenger, F. Wecker, RFID - Leitfaden für die Logistik, Springer (2006), ISBN 978-3-83499188-1

[5] http://www.gs1.org/epcrfid/epc-rfid-uhf-airinterface-protocol/2-0-0, available 03/2015

[6] D. Dobkin, The RF in Rfid: Passive UHF Rfid in Practice, Newnes (2012), ISBN: 0123945836

[7] Courbat J, Briand D, Damon-Lacoste J, Wöllenstein J, de Rooij NF, Evaluation of $\mathrm{pH}$ indicator-based colorimetric films for ammonia detection using optical waveguides, Sensors and Actuators B 143 (2009), 62-70

[8] Courbat J, Briand D, Wöllenstein J, de Rooij NF, Colorimetric gas sensors based on optical waveguides made on plastic foil, Procedia Chemistry 1 (2009): 576-579

[9] C. Peter et al, Low-cost roll-to-roll colorimetric gas sensor system for fire detection, Solid-State Sensors, Actuators and Microsystems Transducers \& Eurosensors XXVII (2013), 26322635, DOI: 10.1109/Transducers.2013.6627346 